\title{
Potentiometric Differentiation of Mono and Macromolecular Thiol within Human Plasma at Carbon Fiber Electrodes
}

\author{
Laura Yonge ${ }^{1}$, Svetlana Gracheva ${ }^{1}$, Shelley J. Wilkins ${ }^{2}$, Callum Livingstone ${ }^{3}$ and James Davis ${ }^{1 *}$ \\ ${ }^{I}$ School of Biomedical and Molecular Sciences, University of Surrey, Guildford, GU2 7XH, UK, ${ }^{2}$ Department of \\ Materials Science, University of Oxford, Parks Road, Oxford, OX1 3PH, ${ }^{3}$ Royal Surrey County Hospital NHS Trust, \\ Guildford, GU2 7XX, UK
}

RECEIVED DATE (automatically inserted by publisher); james.davis@ surrey.ac.uk

\section{Supporting Information}

Electrode Fabrication

A $1 \mathrm{~cm}$ square of pressed carbon fiber matting (E-Tek Inc, USA) and connecting copper foil contact were thermally sandwiched between commercial resin backed polyester laminating pouches (75 micron thick). Two designs were studied - one in which the laminate was pre-punched to reveal a disk (1 $\mathrm{cm}^{2}$ ) of carbon fiber and one where by the matting was completely encapsulated within the laminate. The latter was then drilled to expose a thin, cylindrical layer of severed carbon fibers. The electrodes were then baked in an oven at $100^{\circ} \mathrm{C}$ to ensure the resin securely adheres to the underlying pressed fiber and prevents permeation of the electrolyte within the bulk of the electrode.

\section{Electrode Cell Operation}

All reagents were of the highest grade available and used without further purification. All solutions and subsequent dilutions were prepared daily using deionised water from an Elgastat (Elga, UK) water system and were refrigerated when not in use. Electrochemical measurements were conducted using a Sycopel AWE-10 computer controlled potentiostat. Preliminary investigations were conducted with an initial cell volume of 10 $\mathrm{cm}^{3}$ with the measurements recorded at a temperature of $20^{\circ} \mathrm{C} \pm$ $2^{\circ} \mathrm{C}$. Potentiometric responses were recorded using a conventional two electrode assembly with a commercial silver / silver chloride reference electrode (3M NaCl, BAS Technicol). Unless specified otherwise, the solutions consisted of Britton-Robinson buffer (acetic, boric and phosphoric acids - each at a concentration of $0.04 \mathrm{M}$ and adjusted to $\mathrm{pH} 7$ through the addition of sodium hydroxide).
Potentiometric detection was used as it avoids problems traditionally associated with applying voltammetric techniques to physiological samples. In the latter, the voltammetric signal from the oxidation of ascorbate (and often other constituents such as urate, tyrosine, tryptophan etc) tends to overlap with that of the quinone-thiol conjugate with the loss of resolution compromising the analytical integrity of the measurement. The problems associated with voltammetric/amperometric detection of thiols within physiological fluids have been reviewed by the authors and the reader is directed to references 7 and 8 for more detailed information. The passive nature of the potentiometric signal and specificity for the change in redox potential arising form the quinone-thiol reaction alone provides a significant advantage.

\section{Clinical Trials}

A clinical trial involving 2 non-diabetic control subjects and 2 well controlled (HbA1c $<7.5 \%$ ) type- 2 diabetic subjects, 3 male and 1 female, ranging from 49-67 years of age was conducted. Samples of blood were obtained from the participants and analysed within three hours of extraction. Blood was collected in heparinised gel permeation vaccutainers and then centrifuged at $3000 \mathrm{rpm}$ for 10 minutes. The plasma was withdrawn and used immediately for both electrochemical and spectroscopic analysis. The latter was conducted using established Ellman's Assay protocols with the calibration and analysis data taken at $412 \mathrm{~nm}$.

Both carbon fiber mat and ring were re-used with simple immersion and subsequent rinse in the buffer $(\mathrm{pH} 7)$ solution sufficient to regenerate the electrode performance. There was no degradation in electrode response irrespective of whether they were used in constructing the calibration data or analysing plasma samples. This is corroborated by the RSD values for the clinical measurements quoted in table 1 . In this instance the values refer to three repetitive analyses at the same electrode. 